\title{
Breaking the black-body limit with resonant surfaces
}

\author{
Constantinos A. Valagiannopoulos ${ }^{1, *}$, Constantin R. Simovski ${ }^{2}$, and Sergei A. Tretyakov ${ }^{2}$ \\ 1 Department of Physics, School of Science and Technology, Nazarbayev University, KZ-010000 Astana, Kazakhstan \\ 2 Department of Radio Science and Engineering, School of Electrical Engineering, Aalto University, P.O. Box 13000, \\ Aalto FI-00076, Finland
}

Received 29 August 2016 / Accepted 15 January 2017

\begin{abstract}
The speed with which electromagnetic energy can be wirelessly transferred from a source to the user is a crucial indicator for the performance of a large number of electronic and photonic devices. We expect that energy transfer can be enhanced using special materials. In this paper, we determine the constituent parameters of a medium which can support theoretically infinite energy concentration close to its boundary; such a material combines properties of Perfectly Matched Layers (PML) and Double-Negative (DNG) media. It realizes conjugate matching with free space for every possible mode including, most importantly, all evanescent modes; we call this medium Conjugate Matched Layer (CML). Sources located outside such layer deliver power to the conjugate-matched body exceptionally effectively, impressively overcoming the black-body absorption limit which takes into account only propagating waves. We also expand this near-field concept related to the infinitely fast absorption of energy along the air-medium interface to enhance the far-field radiation. This becomes possible with the use of small particles randomly placed along the boundary; the induced currents due to the extremely high-amplitude resonating fields can play the role of emission "vessels", by sending part of the theoretically unlimited near-field energy far away from the CML structure.
\end{abstract}

Key words: Black-body limit, Conjugate matching, Perfectly Matched Layer (PML), Wireless power transfer.

\section{General principles}

\subsection{Introduction and motivation}

The selection of configurations and materials to achieve a desired distribution of electromagnetic energy in space and time is a general and fascinating topic covering a broad scientific area within electromagnetics and photonics. In particular, how the available energy from a primary source can be very rapidly and suitably delivered to another region of space is an issue with numerous applications. Designs of effective photovoltaic cells $[1,2]$, wireless charging components [3, 4] and light steering subwavelength metasurfaces $[5,6]$ are only a few of the application areas with huge practical interest.

Additionally, one remarkable class of problems where wireless transfer of power and its distribution is crucial concerns understanding, design, and fabrication of electromagnetic absorbers. A thorough overview of potential topologies for ultra-efficient absorbers wrapped into a general theory of thin perfect absorbing layers is provided in [7], where suitable models and designs are given for each of the identified families. Furthermore, so-called perfect metamaterial absorbers have been fabricated with sole use of metallic elements which are experimentally tested with excellent results [8], and are

*e-mail: valagiannopoulos@gmail.com examined in alternative structures $[9,10]$. Other interesting designs in the visible spectrum $[11,12]$, the sub-THz spectrum [13] and at radio frequencies [14] have been reported to exhibit high efficiency combined with broadband features.

It should be stressed that the performance of all the aforementioned absorbing configurations is bounded by the performance of the so-called ideal black body, which completely absorbs all the incident electromagnetic radiation, regardless of the angle of incidence and polarization; the corresponding concept is widely used in thermodynamics, optics, and radio engineering. Several attempts to emulate the response of a black body have been made in acoustics [15] and photonics [16] with some success. However, very recently absorbing structures which break that upper limit posed by perfectly black body have been proposed [17, 18], and they are based on the use of Double-Negative (DNG) uniaxial media which obey the Perfectly Matched Layer (PML) rule. That Conjugate Matched Layer (CML), as we call it, and its variants, would be the major topic of the present work, where alternative excitations are considered. In particular, the proposed CML concept is described in the Section 1.2, while its enormous, super-Planckian absorbing performance is demonstrated in Section 2. The ability of the analyzed component to send a part of the giant near-field power concentrated at its surface, to the far-zone, is examined in Section 3. In particular, we perturb slightly the structure by putting one (Sect. 3.1) 
or multiple (Sect. 3.2) particles in the region with strong background field. Due to the diffuse scattering occurred from the induced currents, the far-field radiation of the device becomes stronger than that of the corresponding ideal black body.

\subsection{Conjugate matched layer (CML) concept}

In order to maximize the power wirelessly delivered from a source to a load, the load should be conjugate matched to the internal impedance of the source. This well-known maximal power principle applied in circuits can be generalized to cover electrically sizable electromagnetic structures. The only difference is that, in the latter case, there are infinitely many channels (modes) for transferring energy; if all of them obey the conjugate-matching principle, the transferred power $P$ is diverging [17]. In particular, the load can be replaced by a semi-infinite half-space filled with a uniaxial medium of relative constituent properties $\left(\varepsilon_{r t}, \mu_{r t}, \varepsilon_{r n}\right)$ and the source by any dipole or multipole placed in the vicinity of the air-medium interface [18]. Considering TM (magnetic field with one sole Cartesian component) illumination (which does not damage the generality), the internal impedance of the source is the one of free space: $Z_{0}=-j \frac{\eta_{0}}{k_{0}} \sqrt{k_{\mathrm{t}}^{2}-k_{0}^{2}}$, where $k_{0}=2 \pi / \lambda_{0}=2 \pi f \sqrt{\varepsilon_{0} \mu_{0}}$ is the free-space wavenumber and $k_{\mathrm{t}}$ is the transverse wavenumber for the direction parallel to the interface ( $t$ stands for transverse and $n$ for normal direction with respect to the surface of the material sample). The symbols $\eta_{0}, \lambda_{0}, \varepsilon_{0}$ and $\mu_{0}$ correspond to the free-space impedance, wavelength, permittivity and permeability, respectively $\left(\mathrm{e}^{+j 2 \pi f \tau}\right.$ time dependence is suppressed, and $f$ is the operational frequency). The TM wave impedance of the uniaxial medium is given by: $Z=-j \frac{\eta_{0}}{k_{0} \varepsilon_{r t}} \sqrt{\frac{\varepsilon_{r t}}{\varepsilon_{r t}} k_{\mathrm{t}}^{2}-\varepsilon_{r t} \mu_{r t} k_{0}^{2}}$, where $\left(\varepsilon_{r t}, \mu_{r t}\right)$ are the transverse and normal permittivities, and $\mu_{r t}$ its transverse permeability. It has been shown [18] that the constituent parameters of the uniaxial medium which can achieve conjugate matching with free space $Z\left(k_{\mathrm{t}}\right)=Z_{0}^{*}\left(k_{\mathrm{t}}\right)$ for every single mode $k_{\mathrm{t}}$, should satisfy the Perfectly Matched Layer (PML) rule [19] but with negative real parts of the material parameters, namely:

$$
\varepsilon_{r t}=\mu_{r t}=\frac{1}{\varepsilon_{r n}}=a-j b, \quad a<0 .
$$

That is why we call such an effective material Conjugate Matched Layer (CML). The ordinary PML just behaves like a perfect "black body" $[16,19]$ absorbing solely the propagating modes; on the contrary, CML additionally fully exploits all the evanescent waves. Note that the parameter $b>0$ represents losses along the transverse direction, which means that a medium defined by (1) is active along the normal direction $\left(\operatorname{Im}\left[\varepsilon_{r n}\right]>0\right)$.

\section{Near-field energy transfer}

If we particularize our research to the test-bed configuration of Figure 1a, namely, a grounded slab excited by a tilted (by the angle $\theta$ ) electric-dipole line source (expression for the incident field of such source can be found e.g. in [20]) at distance $g$ from the interface, we can find in analytical form the electromagnetic power $P$ absorbed in the slab. To better understand the variation of $P$, we consider a slightly perturbed version of the material (1) with $\varepsilon_{r n}=1 / \varepsilon_{r t}-j \delta=1 / \mu_{r t}-j \delta$ (for $\delta=0$, we have the perfect CML of (1)). The absorbed power $P$ can be written as a sum of two terms: one expressing the energy transferred via the propagating modes $P_{\text {prop }}$ (which is not dependent on $a, b, \delta$ ) and another corresponding to the evanescent modes $P_{\text {evan. }}$. If we assume a small perturbation parameter $\delta \rightarrow 0, P_{\text {evan }}$ takes the following form [18]:

$$
\begin{aligned}
P_{\text {evan }} \cong & P_{\text {prop }} \frac{8|a|}{k_{0}^{2} \pi} \int_{k_{0}}^{+\infty} \frac{k_{\mathrm{t}}^{2}\left(k_{\mathrm{t}}^{2}-k_{0}^{2} \sin ^{2} \theta\right)}{\left(k_{\mathrm{t}}^{2}-k_{0}^{2}\right)^{\frac{3}{2}}} e^{-2 g \sqrt{k_{\mathrm{t}}^{2}-k_{0}^{2}}} \\
& \times \frac{\delta}{[1+\operatorname{sgn}(a)]^{2}+\delta^{2}\left[\frac{k_{\mathrm{t}}^{2}\left|\varepsilon_{r}\right|}{2\left(k_{\mathrm{t}}^{2}-k_{0}^{2}\right)}\right]^{2}} d k_{\mathrm{t}} .
\end{aligned}
$$

It is remarkable that for the DNG case $(a<0)$ the aforementioned quantity behaves like $P_{\text {evan }} \sim P_{\text {prop }} / \delta$, which means that it takes very high values when the uniaxial slab exhibits electromagnetic behavior close to that of a perfect CML (1). Furthermore, it should be noted that the sign of the absorbed power $P_{\text {evan }}$ (and accordingly the overall power $\left.P=P_{\text {prop }}+P_{\text {evan }}\right)$ is negative when $\delta<0$; it means that the slab acts as a secondary source and pumps (extremely quickly) energy to the system. In other words, our load (the grounded uniaxial slab) can either absorb or emit energy infinitely fast, if it obeys the rule (1) and simultaneously is slightly lossy $(\delta>0)$ or slightly active $(\delta<0)$, respectively. It should be stressed that the aforementioned separation between activity and passivity is valid for the chosen dipole excitation; for a more general characterization of the medium, we should check the signs of the imaginary part of the supported propagation constant and the real part of the wave impedance. Alternatively, one can check the negative definiteness of the dyadic $\left(\left[\varepsilon_{r}\right]-\left[\varepsilon_{r}\right]^{*}\right)$, where $\left[\varepsilon_{r}\right]$ is the complex permittivity matrix as a criterion for the structure passivity [21]. Specializing to our example case, the perturbed CML medium is passive for the chosen dipole excitation when $\delta>0$ but as far as any possible excitation is concerned, we should take values obeying the inequality $\delta>b /\left(a^{2}+b^{2}\right)$.

Extremely efficient absorption is demonstrated by Figure 2, where the ratio $P / P_{\text {prop }}$ is represented as a function of $a$ for various loss parameters $b>0$. We consider two scenarios: one as in Figure 2a, where the rule (1) is followed (not exactly but with a small $\delta>0$ ) and one as in Figure 2b, where the effective parameters of the medium are all passive, namely $\varepsilon_{r t}=\mu_{\mathrm{rt}}=a-j b$ but $\varepsilon_{r n}=\operatorname{Re}\left[1 / \varepsilon_{r t}\right]=a /\left(a^{2}+b^{2}\right)$. In other words, the first case corresponds to a medium with an active normal component but overall the structure absorbs energy from the source $(0<\delta \rightarrow 0)$ and in the second case we consider a non-active normal component with $\delta=b /\left(a^{2}+b^{2}\right)$. The most dominant characteristic of the two graphs is the switch of $P$ from very low values for $a>0$ (conventional PML "black body" absorption $P_{\text {prop}}$, double positive DPS medium) to extremely high values in the CML regime $(a<0)$. It should be stressed that the nonactive case exhibits 
(a)

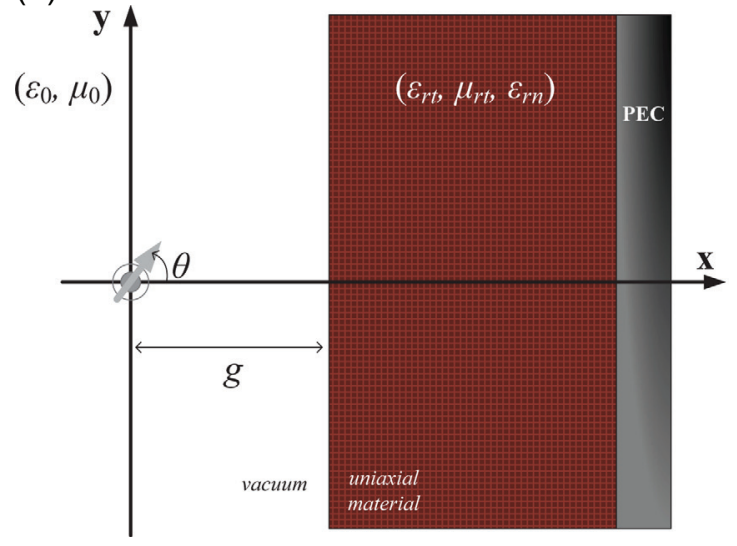

(b)

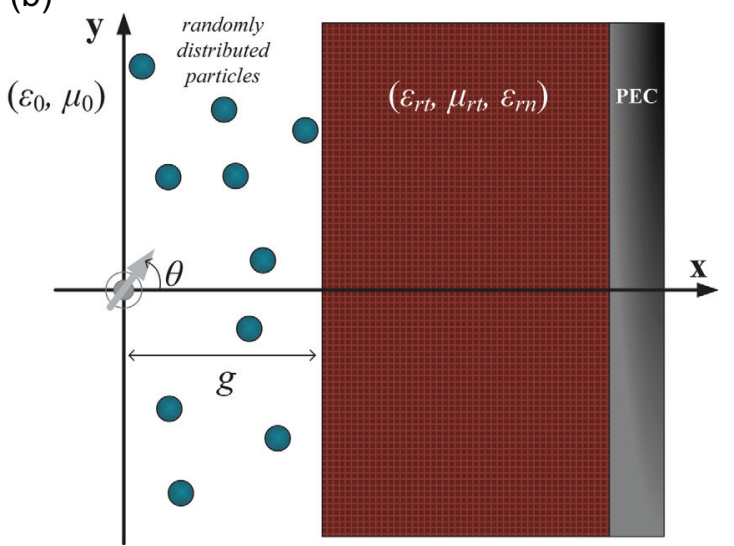

Figure 1. The configurations offering: (a) extremely high absorbing power and (b) very high emitting power.

(a)

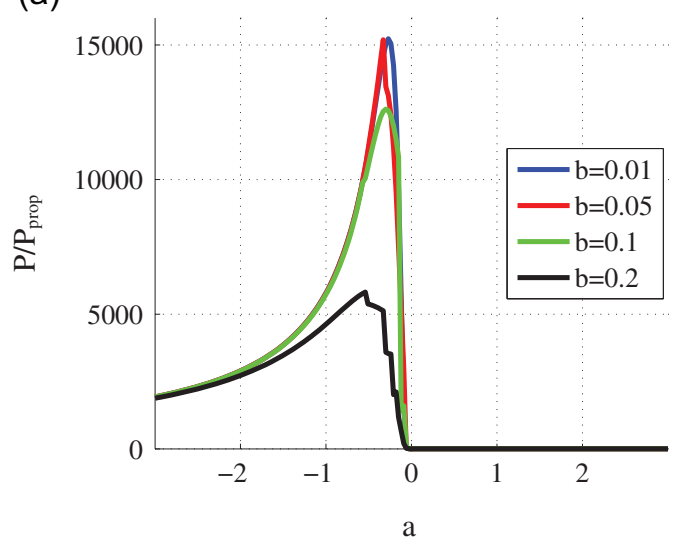

(b)

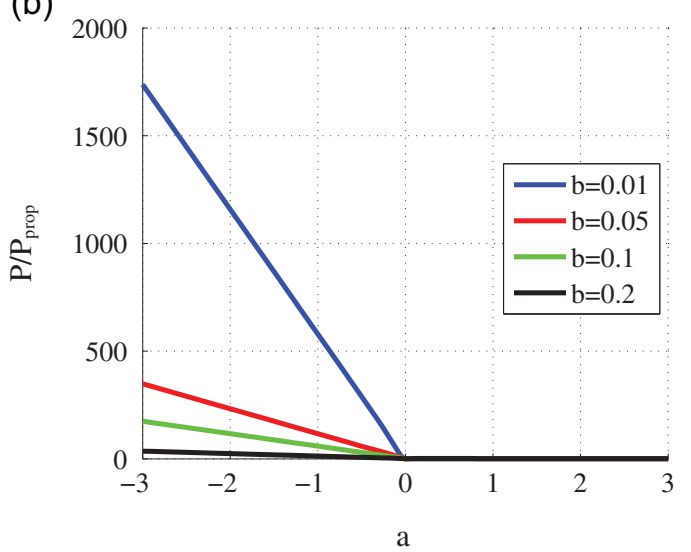

Figure 2. The normalized absorbed power $P / P_{\text {prop }}$ as a function of $a=\operatorname{Re}\left[\varepsilon_{r t}\right]=\operatorname{Re}\left[\mu_{r t}\right]$ for several values of the loss parameter $b$ in: (a) CML passive case and (b) nonactive case.

high absorbing efficiency $\left(P \gg P_{\text {prop}}\right)$, which is, however, smaller than in the CML structure. Furthermore, an interesting feature is the effect of $\delta$ in Figure 2a which makes $P$ to increase substantially for small $|a<0|$; on the contrary, in Figure $2 \mathrm{~b}$, where all the material parameters are passive, the power $P$ is a growing function of $|a<0|$.

\section{Far-field energy transfer}

\subsection{Single particle}

The structure exhibits extremely high efficiency as an emitter, namely has $P_{\text {evan }}, P \rightarrow-\infty$ when choosing a small $|\delta<0|$; however, this does not mean that this huge power travels far away. Due to the nature of evanescent fields which are responsible for the development of $P_{\text {evan, }}$, the resonant fields decay exponentially with the distance from the planar interface. This would not be the case if the slab had a finite size, since its evanescent modes would always have a non-zero propagating factor; however, in this work we confine our research to the simple, analytically solvable configurations of Figure 1. In order to remedy this weakness (of the infinite layer) and enable sending a significant portion of that huge $P$ (which is huge regardless of the choice of the sign of $\delta$ ) to the far-field region, we add a small cylindrical particle in the near field of the structure (close to $x=g$ interface). Furthermore, to understand better the effect of this particle, we consider a simple plane wave excitation instead of the tilted dipole of Figure 1a, namely, an incident $\hat{z}$-polarized magnetic field given by: $H_{\text {inc }}(x, y)=\exp \left[-x \sqrt{k_{\mathrm{t}}^{2}-k_{0}^{2}}-j k_{\mathrm{t}} y\right]$. If the single particle is located at the origin of the coordinate system and is made from a perfect magnetic conductor (a lossless scatterer), the magnetic current $M$ measured in Volt that would be induced along it, normalized by the corresponding current $M_{0}$ in the absence of the CML structure, is written as:

$$
\frac{M}{M_{0}}=\frac{1+e^{-2 g \kappa_{0}\left(k_{\mathrm{t}}\right)} \frac{\varepsilon_{r} \kappa_{0}\left(k_{\mathrm{t}}\right)-\kappa\left(k_{\mathrm{t}}\right)}{\varepsilon_{r} \kappa_{0}\left(k_{\mathrm{t}}\right)+\kappa\left(k_{\mathrm{t}}\right)}}{1+\frac{2 j}{\pi H_{0}^{(2)}\left(k_{0} r\right)} \int_{0}^{+\infty} \frac{e^{-2 g \kappa_{0}(\xi)}}{\kappa_{0}(\xi)} \frac{\varepsilon_{r} \kappa_{0}(\xi)-\kappa(\xi)}{\varepsilon_{r} \kappa_{0}(\xi)+\kappa(\xi)} d \xi},
$$

where $\kappa_{0}\left(k_{\mathrm{t}}\right)=\sqrt{k_{\mathrm{t}}^{2}-k_{0}^{2}}, \kappa\left(k_{\mathrm{t}}\right)=\sqrt{\frac{\varepsilon_{r t}}{\varepsilon_{r n}} k_{\mathrm{t}}^{2}-k_{0}^{2} \varepsilon_{r t} \mu_{r t}}$, and $r$ is the radius of the particle. The notation $H_{0}^{(2)}$ is used for the Hankel function of zeroth order and second type. 
(a)

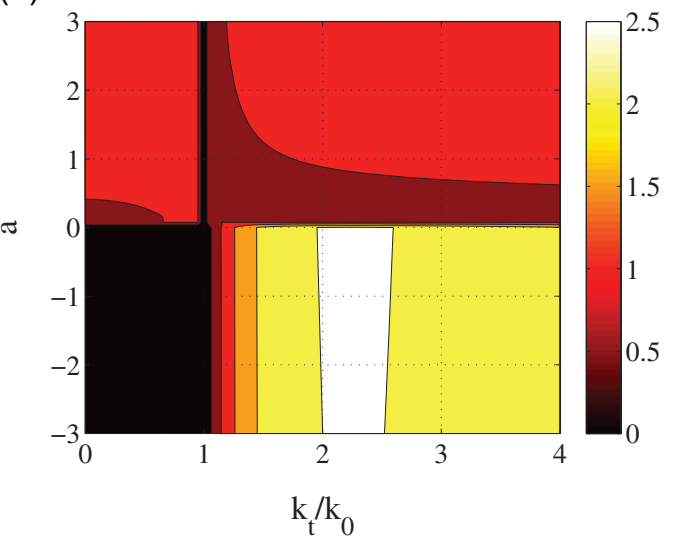

(b)

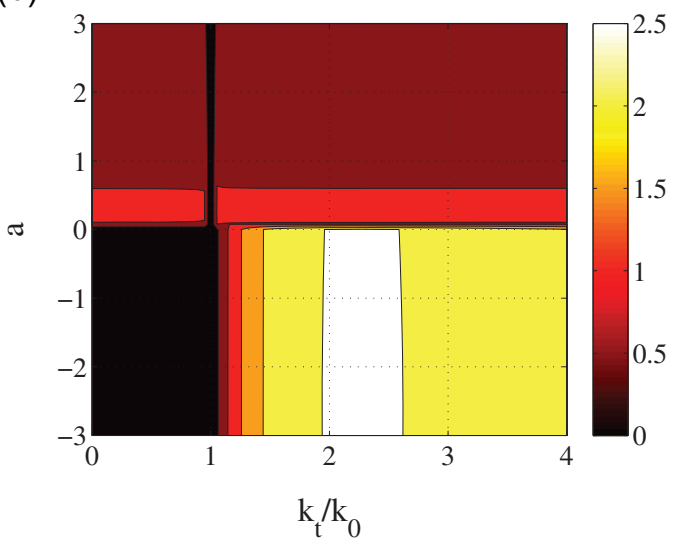

Figure 3. The magnetic current ratio $\left|M / M_{0}\right|$ (perturbed CML case) as a function of the relative transverse wavenumber $k_{\mathrm{t}} / k_{0}$ and the real permittivity and permeability $a$ for: (a) $\delta=0.005>0$ and (b) $\delta=-0.005<0$. Plot parameters: $b=0.2, k_{0} r=0.01, k_{0} g=0.1$.

(a)

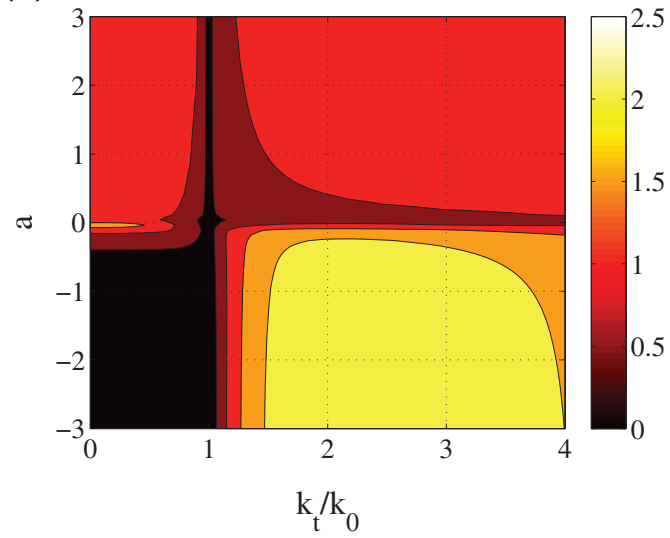

(b)

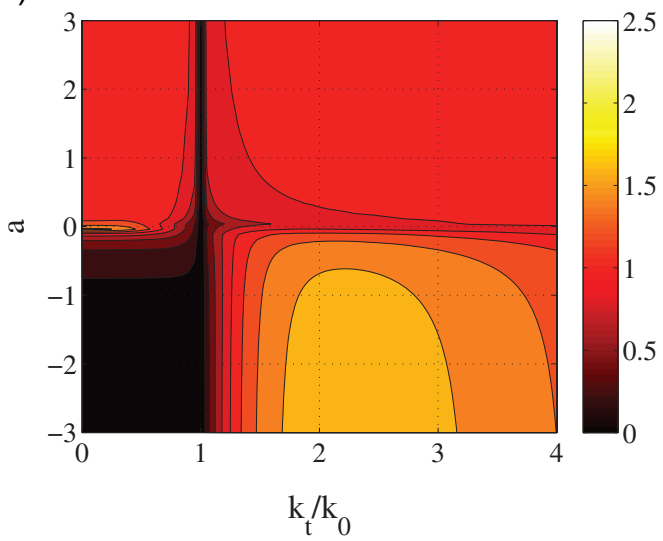

Figure 4. The magnetic current ratio $\left|M / M_{0}\right|$ (nonactive CML case, $\delta=b /\left(a^{2}+b^{2}\right)$ ) as a function of the relative transverse wavenumber $k_{\mathrm{t}} / k_{0}$ and the real permittivity and permeability $a$ for: (a) $k_{0} r=0.01$ and (b) $k_{0} r=0.05$. Plot parameters: $b=0.2, k_{0} g=0.1$.

Expression (3) is obtained by assuming that the thickness $L$ of the slab is infinite: $k_{0} L \rightarrow+\infty$ (the layer behaves like a half space). The choice of the lossless material (magnetic conductor in this example) of the pin does not play a crucial role; it is made on the basis that renders the formulated boundary value problem simpler. Naturally, the type and the size of the particle of course affect the field distributions, but not decisively. Since the background field is very strong in the region, the induced currents along the cylinder will be rather strong; therefore, it will serve the goal of enhancing the radiation regardless of the kind of object we use, as long as the object is electrically small.

In Figure 3 we show the variation of the magnitude of $M / M_{0}$ in contour plot with respect to the relative transverse wavenumber of the incident field $k_{\mathrm{t}} / k_{0}$ and the real part of the transverse permittivity/permeability of the CML medium a. In Figure 3a, the CML structure is selected with $\delta>0$ and we can clearly note that the large enhancement in the current of the radiator happens when the excitation is an evanescent wave $\left(k_{\mathrm{t}}>k_{0}\right)$; on the contrary, for the propagating modes $\left(k_{\mathrm{t}}<k_{0}\right)$, the driving current $M$ is much smaller than in the case of vacuum background $\left(M_{0}\right)$. Furthermore, the switch between the DPS and DNG media (sign of $a$ ), which is indicated by Figure 2 also holds since the variation of the represented quantity for $a>0$ is not significant. In Figure $3 \mathrm{~b}$ we show the results for a CML structure with $\delta<0$ and the distribution of $\left|M / M_{0}\right|$ does not differ substantially from that of Figure $3 \mathrm{a}$. This feature clearly shows that the radiation enhancement is not much dependent on the sign of $\delta$, namely on the overall character (absorbing or emitting) of the structure for the dipole excitation of Figure 1a.

In Figure 4 we regard the nonactive case of the example grounded slab (where $\delta=b /\left(a^{2}+b^{2}\right)$ ) and represent the same quantity $\left|M / M_{0}\right|$ as above on the same map $\left(k_{\mathrm{t}} / k_{0}, a\right)$. In Figure $4 \mathrm{a}$ we consider a pin with the electrical radius $k_{0} r=0.01$. Again we see how much suppressed gets the current $M$ when $a<0$ and propagating waves $\left(k_{\mathrm{t}}<k_{0}\right)$ are used as an excitation and how mild is the variation when $a>0$ which confirms that a DPS slab does not enhance substantially the induced current (and accordingly the radiated power) on the perfectly magnetically conducting cylinder. It is noteworthy that $M$ is increased when the reported unlimited 
(a)

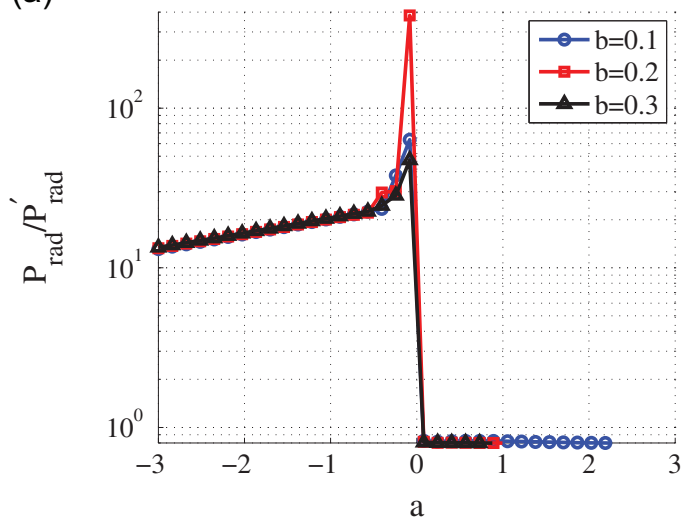

(b)

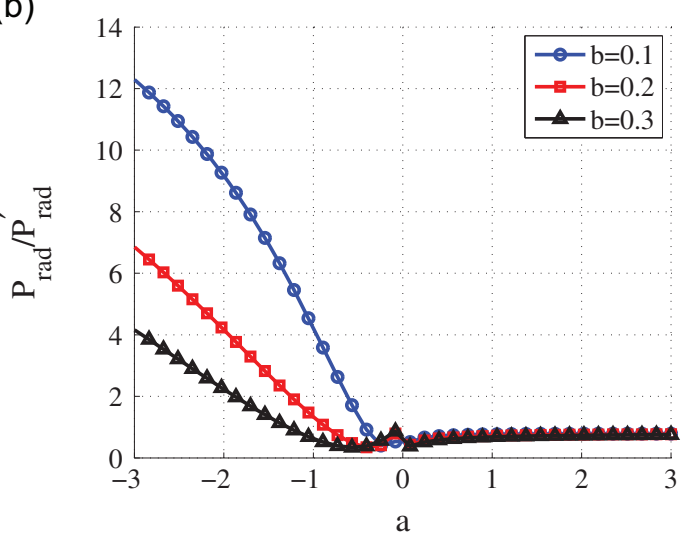

Figure 5. The radiation enhancement ratio $P_{\mathrm{rad}} / P_{\mathrm{rad}}^{\prime}$ as a function of $a=\operatorname{Re}\left[\varepsilon_{r t}\right]=\operatorname{Re}\left[\mu_{r t}\right]$ for several losses $b$ in: (a) CML passive case and (b) nonactive case.

power concentration is developed (DNG and evanescent modes). The same conclusions are drawn from Figure $4 \mathrm{~b}$ where a thicker dipole $\left(k_{0} r=0.05\right)$ is considered. One can observe that $\left|M / M_{0}\right|$ is smaller across the bottom right region of the map compared to Figure 4a; however, that does not necessarily correspond to a lower radiated power since the pin is (five times) more sizeable.

\subsection{Multiple random particles}

By observing the Figures 3 and 4, we remark that the ratio $\left|M / M_{0}\right|$ of the induced currents is not very high, when the structure is excited by a propagating plane wave. The same conclusion is true if we consider as excitation, instead of a plane wave, the dipole of Figure 1a. Reciprocally, if we would use this single pin or a single dipole as a radiating antenna, the presence of a resonant CML close to the small source would not increase radiation into the far zone, since only the evanescent modes will be enhanced. That is why we propose the configuration depicted in Figure $1 \mathrm{~b}$ where multiple pins (acting as "radiation vessels") are located in the vicinity of the air-medium interface. That large number of randomly distributed particles, covering a length comparable or larger than the wavelength and positioned in the near field of the structure are able to couple the evanescent fields with the propagating free-space modes and radiate far away from the interface. The considered boundary value problem can be treated with an integral equation formulation [22-24] which fully describes the wave interactions between the source, the CML slab and the cylindrical particles. In this way, one can evaluate the radiated power in the presence $\left(P_{\text {rad }}\right)$ and the absence $\left(P_{\text {rad }}^{\prime}\right)$ of the particles and accordingly compute the ratio $P_{\text {rad }} / P_{\text {rad }}^{\prime}$ which shows how significant is the radiation enhancement.

The dramatic radiative effect of the particles located in the near field of the CML body is shown in Figure 5, where the parameter $P_{\text {rad }} / P_{\text {rad }}^{\prime}$ is represented as a function of $a$ for various loss levels $b>0$. We consider two cases similar to those of Figure 2 in the presence of numerous electrically small cylinders in the vicinity of the interface. Again the proposed solution works only for DNG media $(a<0)$ where extremely high values of the radiation enhancement ratio are observed for the CML case (Figure 5a). It is noteworthy, that for $a>0$, the radiated far-field is even lower than in the vessel-free structure. Such a result can be explained through partial screening of the propagating modes of the vessel-free structure. In Figure $5 \mathrm{~b}$ of the nonactive scenario, we notice the same behavior of $P_{\text {rad }} / P_{\text {rad }}^{\prime}<1$ for small negative $a$. It can be attributed to out-of-phase far-field responses of the slab and the particles which are of similar magnitudes due to the weak background field as indicated by Figure $2 \mathrm{~b}$. One can observe significant similarities between Figures 2 and 5 , which is natural since both effects have the same physical reason: resonant excitation of surface modes. In particular, we have evanescent modes in both regions which are vanishing away from the interface and their nature is plasmonic. Clearly, we have two adjacent half spaces filled with materials whose permittivity has opposite signs (their real parts); therefore, collective electronic excitation is taking place and surface plasmons are excited $[25,26]$. In contrast with other works [27-29], we do not care much about the spatial distribution of electromagnetic field owed (or not) to surface modes; we use the developed surface waves along the boundary as "containers" of concentrated energy, which is either absorbed by the CML slab (without particles) or emitted in free-space (with particles).

\section{Conclusion}

To conclude, we revisited the recently introduced concept of CML medium (in both its exact and non-active versions), which leads to huge near field enhancement along its interface, and examined the effect of a single particle placed in its vicinity. The particle acts as a radiation vessel and couples the evanescent modes developed on the CML planar surface with cylindrical radiated waves. We report substantial far-field radiation enhancement when multiple randomly placed particles are positioned close to the CML-vacuum interface. The most important limitation of the proposed idea, since it has not been actually implemented, is imposed by the 
difficulties in fabrication of a CML medium. Towards this direction, we are planning the use of fishnet metamaterials (metallic surfaces with holes) or, alternatively, the employment of binary metasurfaces (gratings with two alternating media). In case of success in realizations, it will pave the way for the fabrication of revolutionary, ultra-efficient electronic and photonic designs.

Acknowledgements. Dr. Valagiannopoulos acknowledges target program No. 011503029 "NU-Berkeley strategic initiative in warm-dense matter, advanced materials and energy sources for 2014-2018" from the Ministry of Education and Science of the Republic of Kazakhstan.

\section{References}

1. H.A. Atwater, A. Polman, Plasmonics for improved photovoltaic devices, Nature Materials 9 (2010) 205-213.

2. G. Li, V. Shrotriya, J. Huang, Y. Yao, T. Moriarty, K. Emery, Y. Yang, High-efficiency solution processable polymer photovoltaic cells by self-organization of polymer blends, Nature Materials 4 (2005) 864-868.

3. A. Kurs, A. Karalis, R. Moffatt, J.D. Joannopoulos, P. Fisher, M. Soljacic, Wireless power transfer via strongly coupled magnetic resonances, Science 317 (2007) 83-86.

4. A.P. Sample, D.A. Meyer, J.R. Smith Analysis, Experimental results, and range adaptation of magnetically coupled resonators for wireless power transfer, IEEE Transactions on Antennas and Propagation 58 (2011) 544-554.

5. N.M. Estakhri, A. Alu, Manipulating optical reflections using engineered nanoscale metasurfaces, Physical Review B 89 (2014) 235419.

6. Y. Radi, V.S. Asadchy, S.A. Tretyakov, Tailoring reflections from thin composite metamirrors, IEEE Transactions on Antennas and Propagation 62 (2014) 3749-3760.

7. Y. Radi, C.R. Simovski, S.A. Tretyakov, Thin perfect absorbers for electromagnetic waves: theory, design, and realizations, Physical Review Applied 3 (2015) 037001.

8. N.I. Landy, S. Sajuyigbe, J.J. Mock, D.R. Smith, W.J. Padilla, Perfect metamaterial absorber, Physical Review Letters 100 (2008) 207402.

9. L.L. Spada, L. Vegni, Metamaterial-based wideband electromagnetic wave absorber, Optics Express 6 (2016) 5763-5772.

10. Y.R. Padooru, A.B. Yakovlev, C.S.R. Kaipa, G.W. Hanson, F. Medina, F. Mesa, A.W. Glisson, New absorbing boundary conditions and analytical model for multilayered mushroomtype metamaterials: applications to wideband absorbers, IEEE Transactions on Antennas and Propagation 60 (2012) 5727-5742.

11. X. Chen, L. Liu, P.Y. Yu, S.S. Mao, Increasing solar absorption for photocatalysis with black hydrogenated titanium dioxide nanocrystals, Science 331 (2011) 746-750.

12. C.A. Valagiannopoulos, A. Tukiainen, T. Aho, T. Niemi, M. Guina, S.A. Tretyakov, C.R. Simovski, Perfect magnetic mirror and simple perfect absorber in the visible spectrum, Physical Review B 91 (2015) 115305.
13. B. Wu, H.M. Tuncer, M. Naeem, B. Yang, M.T. Cole, W.I. Milne, Y. Hao, Experimental demonstration of a transparent graphene millimetre wave absorber with $28 \%$ fractional bandwidth at $140 \mathrm{GHz}$, Scientific Reports 6 (2016) 29363.

14. C.A. Valagiannopoulos, S.A. Tretyakov, Symmetric absorbers realized as gratings of PEC cylinders covered by ordinary dielectrics, IEEE Transactions on Antennas and Propagation 62 (2014) 5089-5098.

15. Y.I. Bobrovnitskii, Impedance theory of sound absorption: the best absorber and the black body, Acoustical Physics 52 (2006) 638-647.

16. E.E. Narimanov, A.V. Kildishev, Optical black hole: Broadband omnidirectional light absorber, Applied Physics Letters 95 (2009) 041106.

17. S.I. Maslovski, C.R. Simovski, S.A. Tretyakov, Overcoming black body radiation limit in free space: metamaterial superemitter, New Journal of Physics 18 (2016) 013034.

18. C.A. Valagiannopoulos, J. Vehmas, C.R. Simovski, S.A. Tretyakov, S.I. Maslovski, Electromagnetic energy sink, Physical Review B 92 (2015) 245402.

19. S.D. Gedney, An anisotropic perfectly matched layer absorbing medium for the truncation of FDTD lattices, IEEE Transactions Antennas and Propagation 44 (1996) 1630-1639.

20. C.A. Valagiannopoulos, M.S. Mirmoosa, I.S. Nefedov, S.A. Tretyakov, C.R. Simovski, Hyperbolic-metamaterial antennas for broadband enhancement of dipole emission to free space, Journal of Applied Physics 116 (2014) 163106.

21. C.A. Valagiannopoulos, How non-reciprocal is an effective permittivity matrix?, Microwave and Optical Technology Letters 56 (2014) 9.

22. C.A. Valagiannopoulos, On examining the influence of a thin dielectric strip posed across the diameter of a penetrable radiating cylinder, Progress in Electromagnetics Research C 3 (2008) 203-214.

23. C.A. Valagiannopoulos, S.A. Tretyakov, Theoretical concepts of unlimited-power reflectors, absorbers, and emitters with conjugately matched layers, Physical Review B 94 (2016) 125117.

24. C.A. Valagiannopoulos, Electromagnetic scattering of the field of a metamaterial slab antenna by an arbitrarily positioned cluster of metallic cylinders, Progress in Electromagnetics Research 114 (2011) 55-66.

25. J.M. Pitarke, V.M. Silkin, E.V. Chulkov, P.M. Echenique, Theory of surface plasmons and surface-plasmon polaritons, Reports on Progress in Physics 70 (2007) 1-87.

26. J. Polo, T. Mackay, A. Lakhtakia, Electromagnetic surface waves: a modern perspective, Elsevier, New York, 2013.

27. R. Yang, Y. Hao, An accurate control of the surface wave using transformation optics, Optics Express 20 (2012) 9341.

28. S. Xua, H. Xu, H. Gao, Y. Jianga, F. Yuf, J.D. Joannopoulos, M. Soljacic, H. Chena, H. Sunc, B. Zhang, Broadband surfacewave transformation cloak, Proceedings of the National Academy of Sciences of the United States of America 112 (2015) 7635-7638.

29. L. La Spada, T.M. McManus, A. Dyke, S. Haq, L. Zhang, Q. Cheng, Y. Hao, Surface wave cloak from graded refractive index nanocomposites, Scientific Reports 6 (2016) 29363.

Cite this article as: Valagiannopoulos CA, Simovski CR \& Tretyakov SA: Breaking the black-body limit with resonant surfaces. EPJ Appl. Metamat. 2017, 4, 5 . 